\title{
Dosimetric Impact of Tumor Position and Lung Density Variations in Lung Stereotactic Body Radiotherapy
}

\author{
Hideharu Miura ${ }^{1 *}$, Norihisa Masai ${ }^{1}$, Ryoong-Jin $\mathrm{Oh}^{1}$, Hiroya Shiomi ${ }^{1}$, Kouichi Yamada ${ }^{1}$, \\ Muhammad Nauman Usmani ${ }^{2}$, Toshihiko Inoue ${ }^{1}$ \\ ${ }^{1}$ Miyakojima IGRT Clinic, Osaka, Japan \\ ${ }^{2}$ Department of Medical Physics \& Engineering, Osaka University Graduate School of Medicine, \\ Osaka, Japan \\ Email: *hide-miura@osaka-igrt.or.jp
}

Received December 26, 2013; revised January 20, 2014; accepted February 7, 2014

Copyright (c) 2014 Hideharu Miura et al. This is an open access article distributed under the Creative Commons Attribution License, which permits unrestricted use, distribution, and reproduction in any medium, provided the original work is properly cited. In accordance of the Creative Commons Attribution License all Copyrights (C) 2014 are reserved for SCIRP and the owner of the intellectual property Hideharu Miura et al. All Copyright (C) 2014 are guarded by law and by SCIRP as a guardian.

\begin{abstract}
Purpose of this study was to evaluate the variation of the dose to gross tumor volume (GTV) related to tumor position and lung density for lung stereotactic body radiotherapy (SBRT) using a virtual phantom. The density of the equivalent lung surrounding the GTV (10 mm diameter) was defined as $0.10,0.15,0.25,0.35$, and 0.45 $\mathrm{g} / \mathrm{cm}^{3}$. A planning target volume (PTV) was generated by adding a uniform $8 \mathrm{~mm}$ margin to the internal target volume (ITV). We defined that the $99 \%$ of the GTV should be covered by $100 \%$ of the prescribed dose using Monte Carlo (MC) calculation. The GTV structure was replicated from ITV to the PTV periphery at $1 \mathrm{~mm}$ intervals. Planned dose to the GTV was defined as the predicted dose in the replicated GTV structure. Simulated dose to the GTV was defined as the calculated dose in the replicated GTV structure taking into account the tumor position error. D99 of the planned dose to the GTV at the $8 \mathrm{~mm}$ shift position was $78.1 \%, 81.6 \%, 87.3 \%$, $91.4 \%$ and $94.4 \%$ at equivalent lung densities of $0.10,0.15,0.25,0.35$, and $0.45 \mathrm{~g} / \mathrm{cm}^{3}$, respectively. D99 of the simulated dose to the GTV at the $8 \mathrm{~mm}$ shift position was $96.9 \%, 95.3 \%, 94.2 \%, 95.1 \%$ and $96.3 \%$ at equivalent lung densities of $0.10,0.15,0.25,0.35$, and $0.45 \mathrm{~g} / \mathrm{cm}^{3}$, respectively. Planned dose to GTV is strongly dependent on lung density and tumor position errors, while simulated dose to GTV does not show any significant dependence.
\end{abstract}

\section{KEYWORDS}

\section{Lung Cancer; Stereotactic Body Radiotherapy; Tumor Position Error; Lung Density; Monte Carlo}

\section{Introduction}

Stereotactic body radiotherapy (SBRT) of primary lung cancer has been performed in clinical practice, with accomplishment of local tumor control rates between $80 \%$ and $90 \%$ [1-3]. Dose calculations that take into account the inhomogeneity correction have been recently incorporated into lung SBRT. Further consideration should be given to the dose definition for lung inhomogeneity correction, because the dose distribution within a planning target volume (PTV) for lung SBRT can be less homogeneous, often with significant variation [4-7]. Dose prescriptions defined to enclose the PTV often vary

\footnotetext{
${ }^{*}$ Corresponding author.
}

widely among institutions, ranging from $65 \%$ to $90 \%$ relative to the dose at the isocenter [8-10]. Since these dose definitions are not standardized, comparison of dose prescriptions to the PTV among institutions is impossible. It is essential for lung SBRT to define the new concept of dose prescription. Our previous phantom study showed that lung density affected the dose of PTV rather than the dose of gross tumor volume (GTV) by using Monte Carlo (MC) calculation [7]. Therefore, we defined that $99 \%$ of the GTV should be covered by $100 \%$ of the prescribed dose $(\mathrm{D} 99=100 \%$ ) based on MC calculation for lung SBRT. Respiration-induced tumor motion may result in considerable differences between the planned and actual delivered dose. Our previous study supported the clinical 
acceptability of treatment planning for breathing-induced tumor motion based on the dose prescription defined for the GTV [11].

Radiation therapy is conducted in the face of tumor position errors encountered during treatment delivery, including patient setup error, as well as inter- and intrafractional tumor motion. Tumor position error raises concerns over a dose difference between the planned and the delivered dose. Decreased lung density might need special attention to the decreased dose to the PTV with tumor position error [4-7]. This has been done in order to identify the type of tumor position error that has the largest impact on the reduction in tumor control probability [12]. We modeled different components of tumor position and investigated the impact of tumor position on the dose distribution delivered to the GTV with PTV margins that are clinically applied in our institution. The purpose of the study reported here was to evaluate the variation of the dose to GTV related to tumor position and lung density for lung SBRT using a virtual phantom.

\section{Materials and Methods}

Virtual phantoms were created by using iPlan RT image ver. 4.1.1 (Brainlab, Munich, Germany) with standard volume contouring tools, and appropriate Hounsfield numbers were assigned to the GTV and lung. Figure 1 shows a virtual phantom $\left(20 \times 20 \times 20 \mathrm{~cm}^{3}\right)$ with a GTV

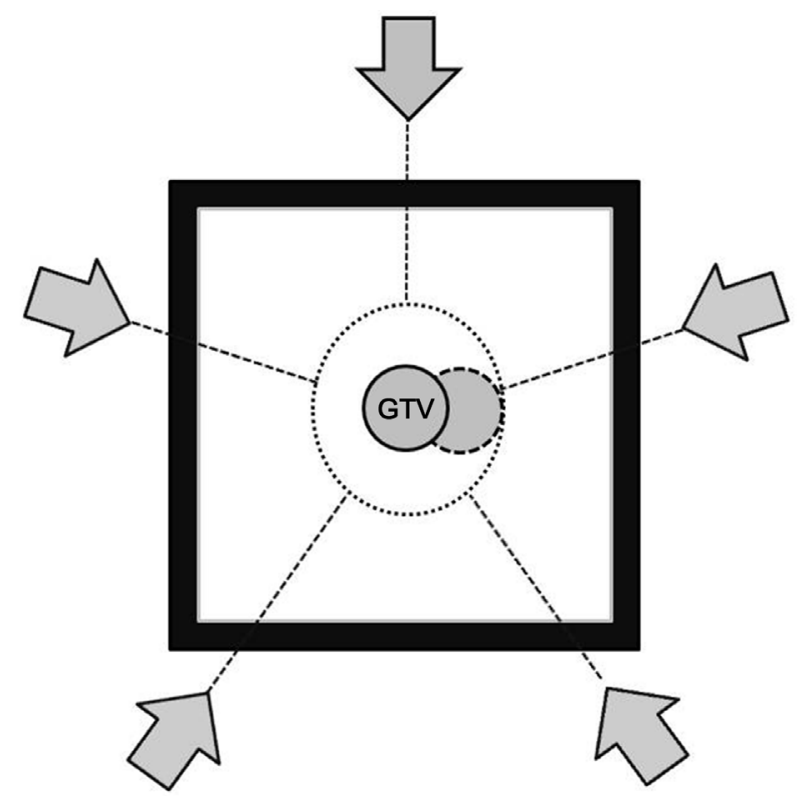

Figure 1. Schematic overview of the foundation model: The centrally located GTV of $10 \mathrm{~mm}$ (grey circle with black solid line) is surrounded by a lung-equivalent material (white) and a $20 \mathrm{~mm}$ wall of water-equivalent material (black). The GTV structure is transposed consistent with the tumor position error (grey circle with black dashed line). Margin to the PTV (dotted line) is $8.0 \mathrm{~mm}$ in all directions. The grey arrows indicate beam directions. of $10 \mathrm{~mm}$ diameter (equivalent water density of 1.0 $\mathrm{g} / \mathrm{cm}^{3}$ ) and a $20 \mathrm{~mm}$ wall of water-equivalent material. The selected GTV size was intended to represent average target dimensions in lung SBRT, and lung density was selected to cover the normal range of lung density. A series of different Hounsfield numbers were assigned to the region representing the lung, namely, $-900,-850$, $-750,-650$ and $-550 \mathrm{HU}$, equivalent to electron densities relative to the electron density of water of $0.10,0.15$, 0.250 .35 and 0.45 , respectively. No additional margin was added for the generation of the clinical target volume (CTV). In our previous study, the average 3D respiratory tumor motion was $2.8 \mathrm{~mm}$ and $6.3 \mathrm{~mm}$ for upper/middle and lower lobes, respectively [11]. Therefore, in current study, the amplitude of tumor motion was set at $5.0 \mathrm{~mm}$ as internal target volume (ITV). In accordance with our routine clinical practice, a uniform $8 \mathrm{~mm}$ margin was added to the ITV to generate a PTV. In this study, we investigated the correlation between tumor position error and dose distribution in various lung densities. For investigation of the dose delivered to the GTV, we replicated the same GTV structure from ITV to the PTV periphery at $1 \mathrm{~mm}$ intervals towards the $\mathrm{x}$ and $\mathrm{z}$ direction.

The plan was calculated using the MC calculation in an iplan RT Dose ver. 4.1.2 (Brainlab, Munich, Germany) treatment planning system with an X-ray Voxel Monte Carlo (XVMC) dose calculation engine. At our institution, a new commercially available MC algorithm, wellcommissioned with 6-MV photon beam, is used for lung SBRT. Commissioning procedure of treatment planning system was given by working groups of the European Society of Radiation Oncology [13]. XVMC has three main stages for the calculation. The first component of the algorithm is a virtual energy fluence model which is used for the modeling of the upper part of the linac treatment head. The second component of the algorithm models the beam collimating system. The third component of the algorithm computes the dose distribution inside the model of the patient. For the MC photon transport simulations, Compton interactions, pair production events and photoelectric absorptions are considered [14-17]. Gantry angles were set at $0^{\circ}, 72^{\circ}, 144^{\circ}, 216^{\circ}$, and $288^{\circ}$, consistent with simple SBRT treatment planning. This plan represents the typical beam set-up used to cover dosage to the GTV. Dose prescription was defined as D99 $=100 \%$ of the GTV as an original plan. Tumor position of each original plan was shifted in x (left-right) and $\mathrm{z}$ (caudal-cranial) directions to evaluate the dosage variations and to estimate the variation of each dosage index with tumor position. These plans were defined as the "tumor-shift plan". The plan was then recalculated using MC calculation while maintaining the same planning parameters, namely for beam arrangement, leaf positions, isocenter, position, and monitor unit, using the 
full MLC geometry simulation “Accuracy Optimized Model" with a spatial resolution of $2 \mathrm{~mm}$ and variance of $2 \%$.

To investigate the variation of dose indices caused by tumor position errors, the following method was used. First, GTV was displaced from the original position to the peripheral of PTV at $1 \mathrm{~mm}$ interval in the original plan. Then, the dose to the PTV (D95) and dose to the regions of each replicated GTV (D99) were obtained as planned doses. Next, the dose distribution was recalculated with each of the replicated GTV in tumor shift plans. In this recalculation of a dose distribution, the replicated GTV was given the density of $1.0 \mathrm{~g} / \mathrm{cm}^{3}$ instead of the original GTV and the dose to the PTV and dose to the replicated GTV were obtained as the simulated doses (Figure 2). Each dose index was obtained for original plan and all tumor-shift plans. Dose-volume histogram (DVHs) analysis of the dose received by the $95 \%$ of target volume of the PTV (D95) and D99 of the GTV were performed.

\section{Results}

Figure 3 shows an example of dose distribution and

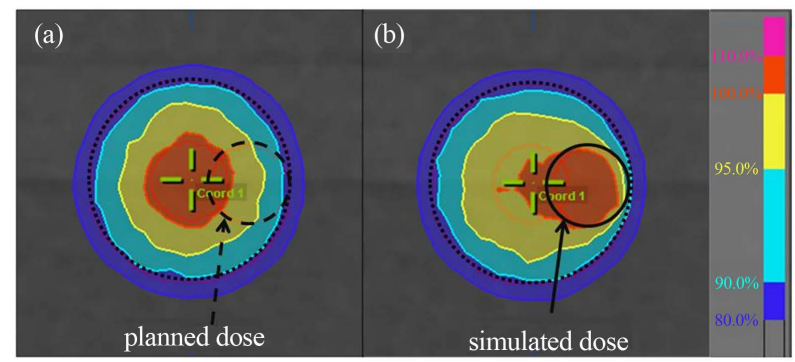

Figure 2. Planned and simulated dose structures: Dashed circle shows the replicated GTV in original plan as planned dose structure and solid black circle shows the replicated GTV with tumor position error as simulated dose structure. PTV is represented by dotted circle.

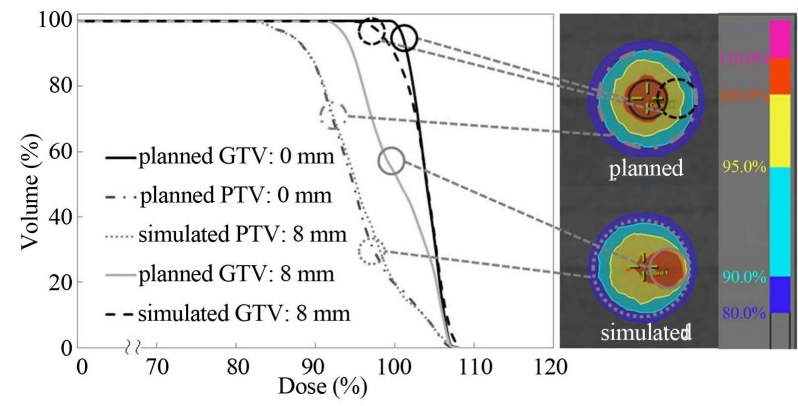

Figure 3. Dose distribution and dose volume histogram for the planned GTV (solid black line), planned PTV (dashed grey line), simulated PTV (dotted grey line) and for the planned GTV (dashed black line) and simulated GTV (solid grey line) with largest tumor position error in a lung density of $0.25 \mathrm{~g} / \mathrm{cm}^{3}$.
DVH for the planned and simulated doses to the GTV at the center and close to the periphery of the PTV in lung density $0.25 \mathrm{~g} / \mathrm{cm}^{3}$. The dose distribution was changed with the GTV position. The planned dose to the GTV decreased when it was placed close to the PTV periphery. The simulated dose to the GTV, placed close to the PTV periphery, was higher than planned dose to the GTV at the same location. The planned and simulated doses to the PTV were almost same.

The D95 of planned dose to PTV relative to the prescribed dose were $77.1 \%, 80.8 \%, 86.2 \%, 90.0 \%$, and $92.6 \%$ with equivalent lung densities of $0.10,0.15,0.25$, 0.35 , and $0.45 \mathrm{~g} / \mathrm{cm}^{3}$, respectively. The Planned dose to the PTV reduced with decreasing lung density. Figure 4 shows the simulated dose to the PTV as a function of tumor position in various lung densities. The planned and simulated doses to the PTV were almost same in various GTV positions at same lung density.

Figure 5 shows the planned dose to the GTV as a function of tumor position in various lung densities. With the shift of the GTV toward the periphery of the PTV, the planned dose to the GTV was decreased. D99 of the planned dose to the GTV at the $8 \mathrm{~mm}$ shift in $\mathrm{x}$ direction were $84.1 \%, 88.1 \%, 92.1 \%, 94.6 \%$ and $96.6 \%$ with equivalent lung densities of $0.10,0.15,0.25,0.35$ and $0.45 \mathrm{~g} / \mathrm{cm}^{3}$, respectively. D99 of planned dose to the GTV

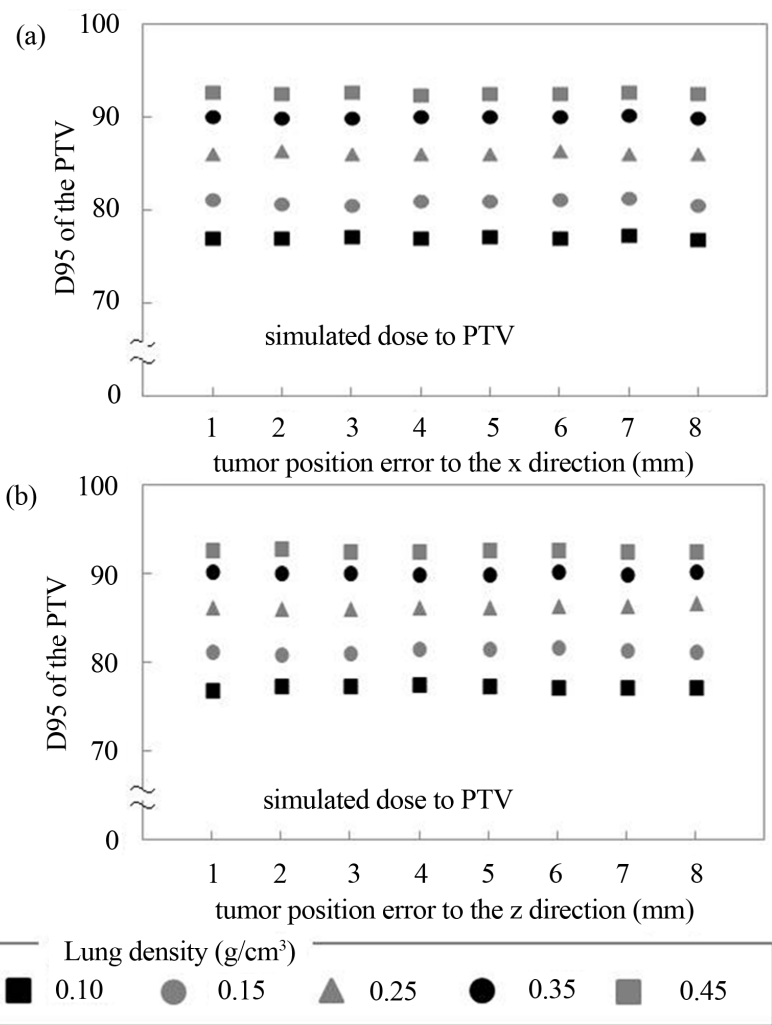

Figure 4. D95 of dose to the PTV as a function of tumor position error to the (a) $\mathrm{x}$ direction and (b) $\mathrm{z}$ direction for five different lung densities. 


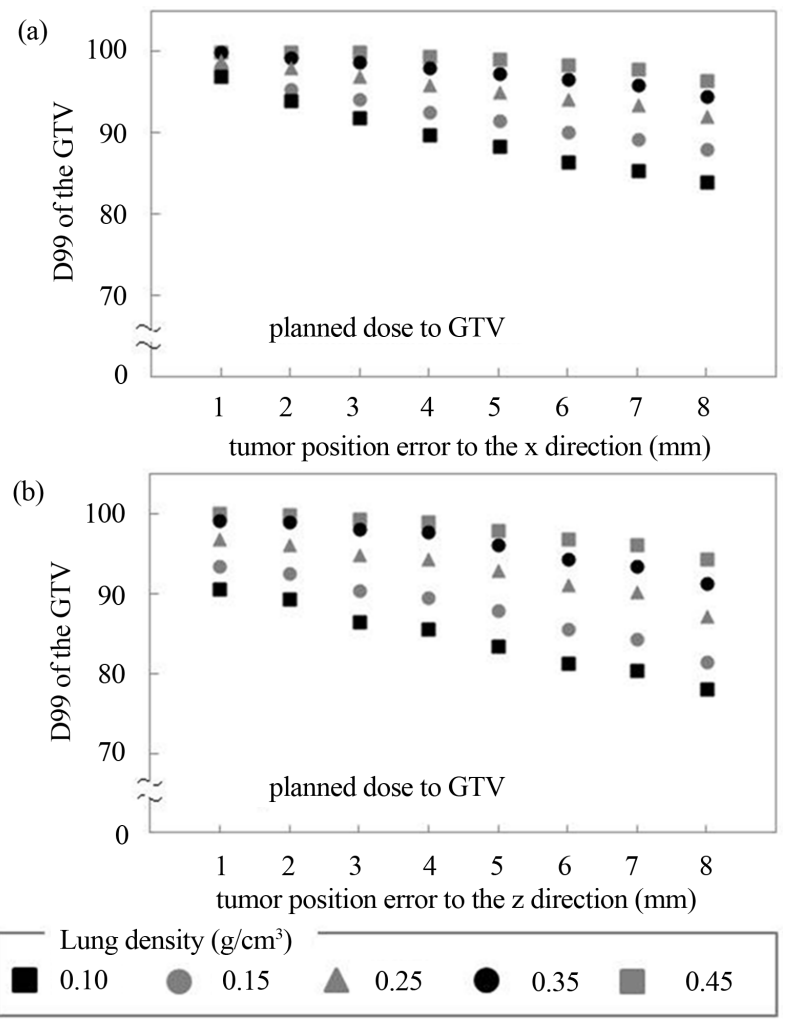

Figure 5. D99 of planned dose to the GTV as a function of tumor position error are shown for five different lung densities: (a) $\mathrm{x}$ direction and (b) $\mathrm{z}$ direction.

at the $8 \mathrm{~mm}$ shift in $\mathrm{z}$ direction were $78.1 \%, 81.6 \%$, $87.3 \%, 91.4 \%$ and $94.4 \%$ with equivalent lung densities of $0.10,0.15,0.25,0.35$ and $0.45 \mathrm{~g} / \mathrm{cm}^{3}$, respectively.

Figure 6 shows the simulated dose to the GTV as a function of tumor position in various lung densities taking into account the tumor position errors. D99 of the simulated dose to the GTV at the $8 \mathrm{~mm}$ shift in $\mathrm{x}$ direction were $97.5 \%, 96.2 \%, 96.1 \%, 97.0 \%$ and $97.5 \%$ with equivalent lung densities of $0.10,0.15,0.25,0.35$ and $0.45 \mathrm{~g} / \mathrm{cm}^{3}$, respectively. D99 of the simulated dose to the GTV at the $8 \mathrm{~mm}$ shift position in z direction were $96.9 \%$, 95.3\%, 94.2\%, $95.1 \%$ and $96.3 \%$ with equivalent lung densities of $0.10,0.15,0.25,0.35$ and $0.45 \mathrm{~g} / \mathrm{cm}^{3}$, respectively.

\section{Discussions}

This study demonstrates the potential of tumor position error to influence the GTV for inhomogeneous dose distributions in a virtual phantom. We observed that the dose to the PTV is reduced with decreasing lung density. Many studies have focused on the effect of variations in lung density and lung geometry on the inhomogeneity correction [4-7]. Many investigators employed MC algorithm to calculate target doses and reported accurate dose calculations in clinical radiotherapy in regions with
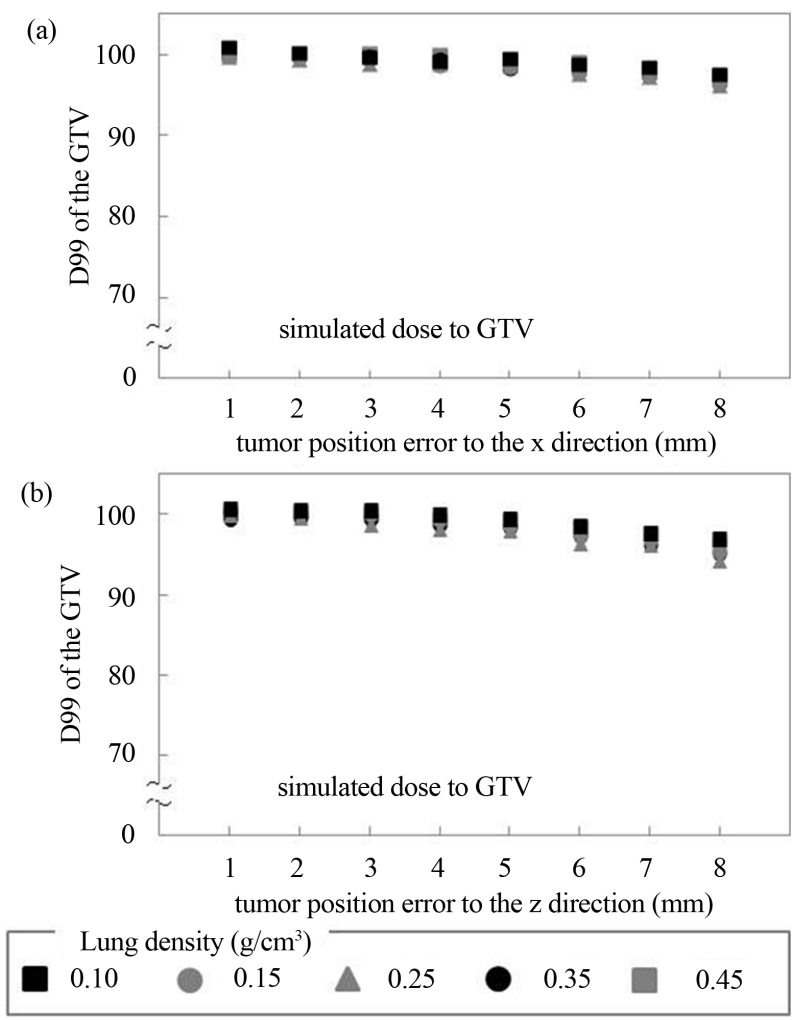

Figure 6. D99 of simulated dose to the GTV as a function of tumor position error are shown for five different lung densities: (a) $\mathrm{x}$ direction and (b) $\mathrm{z}$ direction.

inhomogeneous materials, particularly for lung cancer. The MC calculation is potentially highly accurate as it can faithfully model both electron transport and photon scatter in arbitrary materials [18]. Nakamura et al. showed that the error between the MC calculated planned dose and the measured isocenter dose in moving condition was within $\pm 0.7 \%$ [19]. Krieger and Sauer showed that the Monte Carlo algorithm were very close to the measurements, even in low-density volumes [20].

Our present phantom study suggests that a decreased dose to the planned GTV is an outcome of a lower lung density and a larger tumor position error, particularly in $\mathrm{z}$ direction. Only the coplanar beam was used in this study. Hence, the dose coverage on the superior and inferior target volume was slightly degraded. In clinical practice, this reduction will be much smaller since multiple noncoplanar beams will generally be used. Planned dose to the GTV at lung density of $0.10 \mathrm{~g} / \mathrm{cm}^{3}$ was approximately $20 \%$ lower than prescribed dose in large tumor position error. Simulated dose to the GTV was more stable than planned dose. Owing to scatter conditions, greater doses were absorbed in the solid tumor than in the surrounding lung [7]. We applied a relatively large tumor position error of $8 \mathrm{~mm}$ and found a maximum dose difference of $5 \%$ compared to the prescribed dose. It should be stressed that this situation represents a maximum dose 
reduction in worst scenario. Wang et al. reported that setup variations of approximately 2 - $3 \mathrm{~mm}$ were observed even with cone-beam CT in 50 patients undergoing SBRT [21]. The larger tumor position error is less likely to occur.

A sufficient PTV should allow inter-fractional variation within the defined security margins without a significant decrease in the dose to the CTV [22]. The dose definition is generally prescribed at the PTV, and each institution has its own dose prescription protocol regarding the prescription isodose for lung SBRT [8-10]. If the dose prescription is defined to the PTV, dose to the GTV showed large variations [23], which may lead to a negative effect on tumor local control probability. The International Commission on Radiation Units and Measurements (ICRU) reported that the concept of a PTV might be utilized in unconventional ways to ensure that the prescribed absorbed dose is delivered to the CTV in the future [24].

\section{Conclusion}

In conclusion, we evaluated that tumor position errors and lung densities have a dominant impact on the dose delivered to a lung tumor. We found that planned dose to GTV is strongly dependent on lung density and tumor position errors, while simulated dose to GTV does not show any significant dependence. For clinically representative values of tumor position error and currently applied PTV margins of $8 \mathrm{~mm}$, an inhomogeneity is observed in dose distribution over the PTV, resulting in a maximum drop of approximately 20\%. However, the actual dose to the GTV was about 5\% lower than prescription dose. Dose prescription to the GTV showed sufficient dosage when the GTV was located in a peripheral position of the PTV. This study supports the acceptability of treatment planning based on the dose prescription defined to the GTV.

\section{Conflict of Interest}

None.

\section{REFERENCES}

[1] Y. Nagata, J. Wulf, I. Lax, et al., "Stereotactic Radiotherapy of Primary Lung Cancer and Other Targets: Results of Consultant Meeting of the International Atomic Energy Agency," International Journal of Radiation Oncology* Biology*Physics, Vol. 79, No. 3, 2011, pp. 660-669.

[2] H. Onishi, T. Araki, H. Shirato, et al., "Stereotactic Hypofractionated High-Dose Irradiation for Stage I NonSmall Cell Lung Carcinoma," Cancer, Vol. 101, No. 7, 2004, pp. 1623-1631. http://dx.doi.org/10.1002/cncr.20539

[3] Y. Nagata, K. Takayama, Y. Matsuo, et al., "Clinical
Outcomes of a Phase I/II Study of 48 Gy of Stereotactic Body Radiotherapy in 4 Fractions for Primary Lung Cancer Using a Stereotactic Body Frame,” International Journal of Radiation Oncology*Biology*Physics, Vol. 63, No. 5, 2005, pp. 1427-1431.

[4] L. R. Aarup, A. E. Nahum, C. Zacharatou, et al., "The Effect of Different Lung Densities on the Accuracy of Various Radiotherapy Dose Calculation Methods: Implications for Tumour Coverage," Radiotherapy \& Oncology, Vol. 91, No. 3, 2009, pp. 405-414. http://dx.doi.org/10.1016/j.radonc.2009.01.008

[5] G. X. Ding, D. M. Duggan, B. Lu , et al., "Impact of Inhomogeneity Corrections on Dose Coverage in the Treatment of Lung Cancer Using Stereotactic Body Radiation Therapy," Medical Physics, Vol. 34, No. 7, 2007, pp. 2985-2994. http://dx.doi.org/10.1118/1.2745923

[6] N. C. van der Voort van Zyp, M. S. Hoogeman, S. van de Water, et al., "Clinical Introduction of Monte Carlo Treatment Planning: A Different Prescription Dose for Non-Small Cell Lung Cancer according to Tumor Location and Size,” Radiotherapy \& Oncology, Vol. 96, No. 1, 2010, pp. 55-60. http://dx.doi.org/10.1016/j.radonc.2010.04.009

[7] H. Miura, N. Masai, R. Oh, et al., "Clinical Introduction of Monte Carlo Treatment Planning for Lung Stereotactic Body Radiotherapy,” Journal of Applied Clinical Medical Physics, Vol. 15, No. 1, 2014, pp. 38-48.

[8] M. Guckenberger, J. Wilbert, T. Krieger, et al., "FourDimensional Treatment Planning for Stereotactic Body Radiotherapy," International Journal of Radiation Oncology*Biology*Physics, Vol. 69, No. 1, 2007, pp. 276285.

[9] P. Baumann, J. Nyman, M. Hoyer, et al., "Outcome in a Prospective Phase II Trial of Medically Inoperable Stage I Non-Small Cell Lung Cancer Patients Treated with Stereotactic Body Radiotherapy,” Journal of Clinical Oncology, Vol. 27, No. 20, 2009, pp. 3290-3296. http://dx.doi.org/10.1200/JCO.2008.21.5681

[10] S. W. Lee, E. K. Choi, H. J. Park, et al., "Stereotactic Body Frame Based Fractionated Radiosurgery on Consecutive Days for Primary or Metastatic Tumors in the Lung,” Lung Cancer, Vol. 40, No. 3, 2003, pp. 309-315. http://dx.doi.org/10.1016/S0169-5002(03)00040-0

[11] H. Miura, N. Masai, R. J. Oh, H. Shiomi, J. Sasaki and T. Inoue, "Approach to Dose Prescription of the Gross Tumor Volume for Lung Cancer with Respiratory Tumor Motion,” Journal of Radiation Research, Vol. 54, No. 1, 2013, pp. 140-145. http://dx.doi.org/10.1093/jrr/rrs054

[12] A. E. Nahum and B. Sanchez-Nieto, "Tumour Control Probability Modelling: Basic Principles and Applications in Treatment Planning,” Physica Medica, Vol. 17, No. S2, 2001, pp. 13-23.

[13] ESTRO Booklet No. 7, "Quality Assurance of Treatment Planning Systems,” Practical Examples for Non-IMRT Photon Beams, 2004. http://estro-education.org/publications/Documents/Bookle t_n7-QAorTPS.pdf

[14] M. Fippel, "Fast Monte Carlo Dose Calculation for Photon Beams Based on the VMC Electron Algorithm," 
Medical Physics, Vol. 26, No. 8, 1999, pp. 1466-1475. http://dx.doi.org/10.1118/1.598676

[15] M. Fippel, F. Haryanto, O. Dohm, F. Nüsslin and S. Kriesen, "A Virtual Photon Energy Fluence Model for Monte Carlo Dose Calculation,” Medical Physics, Vol. 30, No. 3, 2003, pp. 301-311. http://dx.doi.org/10.1118/1.1543152

[16] M. Fippel, "Efficient Particle Transport Simulation through Beam Modulating Devices for Monte Carlo Treatment Planning," Medical Physics, Vol. 31, No. 5, 2004, pp. 1235-1242. http://dx.doi.org/10.1118/1.1710734

[17] I. Kawrakow, M. Fippel and K. Friedrich, “3D Electron Dose Calculation Using a Voxel Based Monte Carlo Algorithm (VMC),” Medical Physics, Vol. 23, No. 4, 1996, pp. 445-457. http://dx.doi.org/10.1118/1.597673

[18] Task Group No. 65, the Radiation Therapy Committee of the American Association of Physicists in Medicine, “Tissue Inhomogeneity Corrections for Megavoltage Photon Beams,” Medical Physics Publishing, Madison, 2004.

[19] M. Nakamura, Y. Miyabe, Y. Matsuo, et al., "Experimental Validation of Heterogeneity-Corrected Dose-Volume Prescription on Respiratory-Averaged CT Images in Stereotactic Body Radiotherapy for Moving Tumors,” Medical Dosimetry, Vol. 37, No. 1, 2012, pp. 20-25. http://dx.doi.org/10.1016/j.meddos.2010.12.007
[20] T. Krieger and Q. A. Sauer, "Monte Carlo-Versus Pencil-Beam-/Collapsed-Cone-Dose Calculation in a Heterogeneous Multilayer Phantom,” Physics \& Medicine in Biology, Vol. 50, No. 5, 2005, pp. 859-868. http://dx.doi.org/10.1088/0031-9155/50/5/010

[21] Z. Wang, J. W. Nelson, S. Yoo, et al., "Refinement of Treatment Setup and Target Localization Accuracy Using Three-Dimensional Cone-Beam Computed Tomography for Stereotactic Body Radiotherapy,” International Journal of Radiation Oncology*Biology*Physics, Vol. 73, No. 2, 2009, pp. 571-577.

[22] ICRU, "Prescribing, Recording, and Reporting Photon Beam Therapy,” ICRU Report Volume 50, International Commission on Radiation Units and Measurements, Bethesda, 1993.

[23] H. Miura, N. Masai, R. J. Oh, et al., "Dosimetric Comparison of Different Prescription Modes in Lung Stereotactic Body Radiation Therapy," International Journal of Medical Physics, Clinical Engineering and Radiation Oncology. (In Press)

[24] V. Gregoire, T. R. Mackie, W. D. Neve, et al., "Prescribing Recording and Reporting Photon-Beam IntensityModulated Radiation Therapy (IMRT)," Journal of the ICRU, Vol. 10, No. 1, 2010, pp. 1-112. 